\title{
The anti-inflammatory effects of polyphenols on human adipocytes and innate immune cells isolated from visceral fat
}

\author{
B. Scazzocchio ${ }^{1}$, M. Del Corno ${ }^{1}$, R. Vari $^{1}$, C. Filesi ${ }^{1}$, F. Galvano ${ }^{2}$, M. D'Archivio ${ }^{1}$, M. Fiani ${ }^{1}$, \\ P. Sestili ${ }^{1}$, R. Masella ${ }^{1}$ and S. Gessani ${ }^{1}$ \\ ${ }^{1}$ Istituto Superiore di Sanità, Viale R. Elena, 299, Rome, Italy and ${ }^{2}$ University of Catania, Italy
}

Obesity is now recognised as a low-grade inflammatory condition, the so-called 'metabolic' inflammation, leading to chronic activation of the innate immune system. Although the cascade of molecular events linking over-nutrition, immune system activation and obesity-linked dysfunctions remains poorly understood, a main role has been suggested for insulin-resistance and inflammation. Recently, much attention has been paid to bioactive food components, such as polyphenols, exhibiting therapeutic potential on the obesity-related dysfunctions induced by alterations of pathways critical in the control of inflammation within the adipose tissue.

We have developed an ex-vivo model of human adipocytes and immune cells isolated from the omental fat of normal weight, overweight and obese individuals, allowing to test the efficacy of anti-inflammatory polyphenols such as cyanidin 3-glucoside (C3G) and its main metabolite protocatechuic acid (PCA) in the prevention/attenuation of the obesity-associated inflammation and immune dysfunctions.

Omental biopsies were obtained from 10 patients undergoing abdominal surgery for benign conditions and used for the isolation of adipocytes by collagenase digestion, and immune cells by Ficoll gradient of stromo-vascular fraction. The isolated adipocytes expressed leptin and adiponectin and responded to insulin stimulation. Omental immune cells represented a heterogeneous population in which myeloid dendritic cell (DC)-like cells were identified by FACS analysis as cells highly positive for CD11c and HLA-DR, but negative for a cocktail of lineage markers and by immunostaining of CD1a and DC-SIGN in fixed visceral fat sections. To define the preventive effect of selected polyphenols on cells of the immune system, preliminary studies have been carried out with blood monocyte-derived DCs (MDDCs). We have shown that adipocytes and MDDCs took up PCA (Fig. 1) and responded to C3G and PCA treatment by downmodulating the secretion of some pro-inflammatory cytokines. Specifically, both the polyphenols reduced the basal secretion of TNF- $\alpha$ and IL- 6 by omental adipocytes, while PCA reduced the LPS-stimulated IL-6 and CCL2 production, leaving unchanged the secretion of IL-12, by MDDCs (Table 1). Moreover, these compounds affected glucose transport machinery in omental adipocytes by increasing glucose uptake $(60 \%$ and $40 \%$ with $\mathrm{C} 3 \mathrm{G}$ and PCA, respectively) and stimulating GLUT4 translocation to the plasma membrane (50\% and $110 \%$ with $\mathrm{C} 3 \mathrm{G}$ and PCA, respectively) (Fig. 2).

Overall these results could allow us to define new nutritional strategies for the prevention and therapy of obesity-associated inflammation and immune dysfunctions.

Table 1. Anti-inflammatory effects of polyphenols

\begin{tabular}{|c|c|c|c|c|c|c|c|}
\hline & \multicolumn{3}{|c|}{ Adipocytes } & \multicolumn{4}{|c|}{ MDDCs } \\
\hline & \multirow[b]{2}{*}{ CTR } & \multirow[b]{2}{*}{$\mathrm{C} 3 \mathrm{G}$} & \multirow[b]{2}{*}{ PCA } & \multicolumn{2}{|c|}{- LPS } & \multicolumn{2}{|c|}{+ LPS } \\
\hline & & & & CTR & PCA & CTR & PCA \\
\hline TNF- $\alpha$ & 191 & 90 & 75 & & & & \\
\hline IL-6 & 772 & 287 & 301 & $10 \pm 1$ & $191 \pm 8$ & $3726 \pm 29$ & $2246 \pm 65$ \\
\hline CCL2 & & & & $15 \pm 1$ & $20 \pm 12$ & $1161 \pm 184$ & $279 \pm 25$ \\
\hline IL-12 & & & & $33 \pm 1$ & $40 \pm 8$ & $568 \pm 29$ & $711 \pm 52$ \\
\hline
\end{tabular}

C3G $(50 \mu \mathrm{M})$ and PCA $(100 \mu \mathrm{M})$ reduced the basal secretion of TNF- $\alpha$ and IL-6 by omental adipocytes. PCA reduced the LPS-stimulated production of IL-6 and CCL2, while the secretion of IL-12 was unaffected, by MDDCs. Data are presented as means \pm SD of duplicated wells from one representative of five independent experiments.
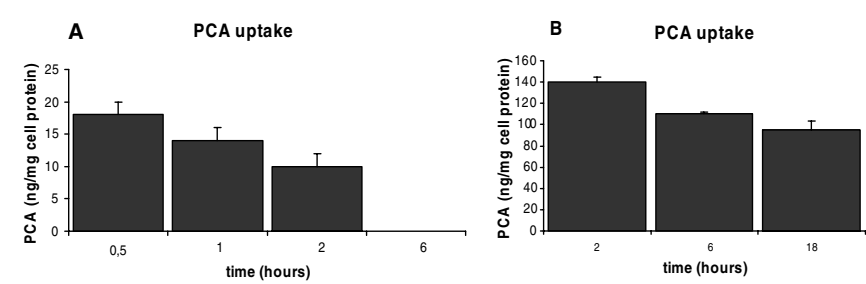

Fig. 1. Polyphenols uptake in adipocytes and MD-DCs. Time-course determination of $100 \mu \mathrm{M}$ PCA uptake measured by HPLC in human adipocytes (A) and MD-DCs (B). Concentrations are reported as $\mathrm{ng} / \mathrm{mg}$ cell proteins. Values are presented as means \pm SD of two experiments performed in duplicate.

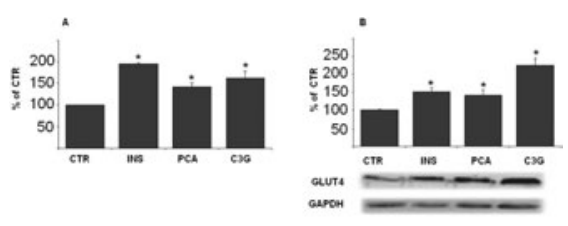

Fig. 2. Insulin-mimetic effect of polyphenols in omental adipocytes. A 2-deoxyglucose (2-DG) uptake in cells treated with $50 \mu \mathrm{M} \mathrm{C} 3 \mathrm{G}$ and $100 \mu \mathrm{M}$ PCA. Data represent mean \pm SEM percentage of incorporated radioactivity with respect to control cells. B. Immunoblotting analysis of plasma membrane GLUT4 in cells treated with $50 \mu \mathrm{M}$ C3G, $100 \mu \mathrm{M}$ PCA or $0,1 \mu \mathrm{M}$ insulin. Data are shown as mean \pm SEM percentage of optical density with respect to control cells. Results were normalized to GAPDH protein content. 